\title{
Modelling and Simulation of the passive Structure of a 5-Axis-Milling Machine with rigid and flexible bodies for evaluating the static and dynamic behaviour
}

\author{
Michael Schneider, B.Eng. ${ }^{1}$ Prof. Anton Haumer ${ }^{1} \quad$ Dipl.-Ing. Rupert Köckeis ${ }^{2}$ \\ ${ }^{1}$ Faculty of Electrical Engineering and Information Technology , OTH Regensburg, 93053 Regensburg, \\ michael. schneiderast. oth-regensburg.de, anton. haumeraoth-regensburg.de \\ ${ }^{2}$ Department of Electrical Engineering, MAX STREICHER GmbH \& Co. KG aA , 94469 Deggendorf, \\ rupert. koeckeisestreicher.de
}

\begin{abstract}
Most of the mechanical simulations for industrial usage are done by finite element (FE-) analysis. Milling machines are mechatronic systems, combining electrical, mechanical and control components for machining certain materials. Modelica provides a powerful and strong tool to simulate different physical areas in one model. For this usage a mechanical model of a 5-Axis-Milling Machine is implemented with rigid and flexible bodies. Specific attention will be paid to which components can be modelled as rigid bodies without significant deviation in accordance to the real behaviour of the machine. Two classes of implementing flexible bodies in multi body systems are given by the Flexible Bodies Library, advantages and disadvantages of both classes will be evaluated. At the end a comparision of the static and dynamic behaviour of the passive structure of the model in contrast to a FE-analysis is given.
\end{abstract}

Keywords: milling machine, flexible body, multibody system, clay modelling

\section{Introduction}

High Speed Cutting (HSC) Machines are present in different technical areas today. The automotive sector uses HSC-Machines for editing clay models of vehicles to improve the design and the aerodynamic behaviour. For this usage high performance and very high precision is required. The validation of mechanical improvements on existing machines is expensive and time consuming. On the other hand FE-Models can not describe the whole mechatronic system, because the exact influence of the electrical drive train in mechanic models is described insufficiently. For this reason a multyphysical model with an electrical, a mechanical and a control system model has to be created. The mechanical model should describe the behaviour of the passive structure in a good approximation. A time efficient model of the machine consisting of rigid and flexible parts without major deviations is developed. During the modelling process several modelling issues have to be solved with particular models.

\section{Structure of the Machine}

Figure 1 shows a front view of the milling machine with its axis designation and its initial frame in the top right corner. Machine constructions where all linear movement

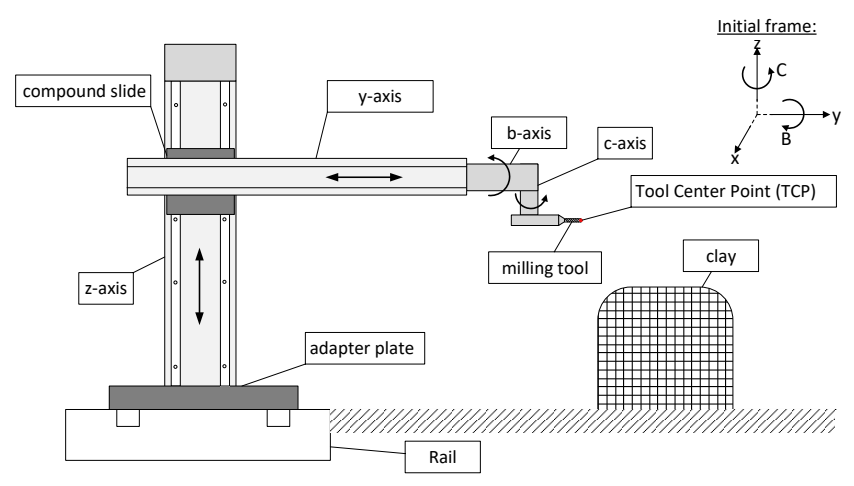

Figure 1. Structure of the milling Machine

axes are facing the milling tool side are called travelling column machines. The considered machine has a horizontal tool spindle, the end of the milling tool is called "Tool Center Point" (TCP). The three main axes ( $\mathrm{x}-, \mathrm{y}-$ and $\mathrm{z}-$ axis) realize linear movements in the cartesian space, two minor axis (b- and c-axis) enable rocking and rolling motions of the TCP. All of the three linear movements are equipped with linear guides for stiff transition between the movable and the fixed part of the machine. A compound slide enables movements in $y$ - and z-direction. At the end of the $y$-axis a milling head is mounted. The linear axes are driven by short stator linear motors, the rocking and the rolling movement is achieved by synchronous motors. An additional synchronous motor drives the milling tool with a very high speed to reduce the cutting force at the TCP. A classification of the different mechanical machine parts is useful. Components with small measurements and high rigidity compared to large machine parts are called "secondary machine parts", large components with low rigidity are called "main machine parts". 


\section{Modelling of the secondary machine parts}

\subsection{Linear guidances at machine tools}

Linear guidances are used to achieve relative movements between fixed and movable parts of machine tools and expose high static, dynamic and thermal stiffness. Furthermore they should show very high dynamical accuracy and low wear running. A classification in two components is usual, a guide carriage mounted at the movable part of the machine and a guide rail at the fixed machine part. The whole carriage-rail system has flexibility in three directions and could therefore deform as a result of a pulling, compressive or a shear force. Modelling this component with flexible elements would terminate in long simulation times due to the incidence of this component in the whole structure. For the purpose of simplification this component could be modeled with rigid bodies if the flexible transition between carriage and rail is modeled in an other more handable way(Queins, 2005, p. 50). But to represent the flexibility of the whole carriage-rail system, that has a significant influence on the static and dynamic behaviour of the structure, a possible replacement for a flexible body model of the carriage-rail system is given at Figure 2. The

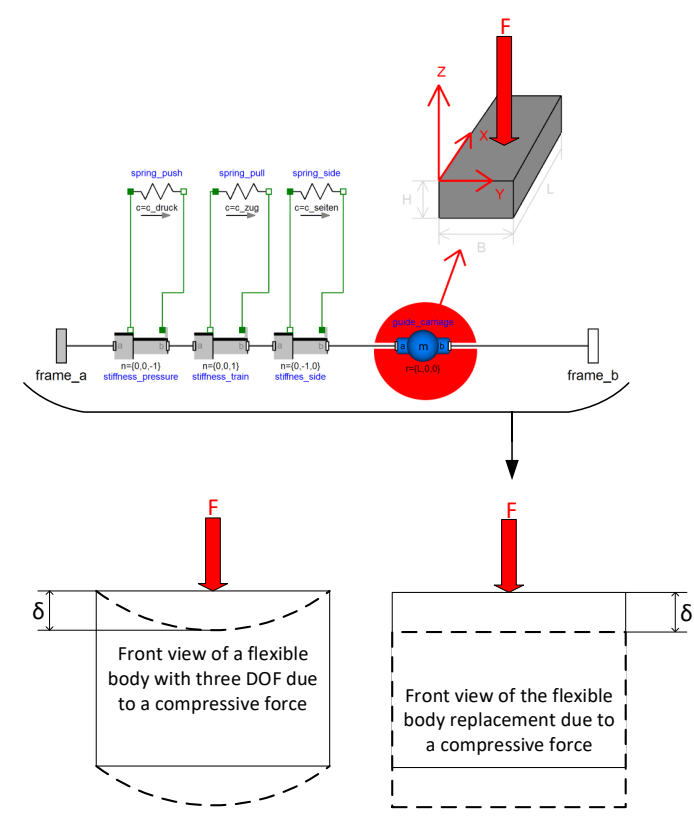

Figure 2. Replacement for a flexible body system by a rigid body system for a carriage-rail component

values of the spring stiffnesses can be calculated through a linearization of the spring charactersistic curve of the carriage-rail system. This curves show a progressive or a degressive characteristic of the carriage-rail system but in a good approximation it can be linearized at the operating point(Queins, 2005, p. 50). The spring characteristic can be assumed as linear subsequently to the large stiffness of the carriage-rail system compared to other more flexible machine components.

\subsection{Model of the adapter plate and the com- pound slide}

The adapter plate connects the moving part of the machine with the machine base also called "rail". This connection is obtained by a linear recirculating roller bearing, which is especially used for longitudinal guides that provides high stiffnes of guidance systems. The adapter plate consists of two linear guidance systems with four guide carriages that are mounted at a mechanical cast component. Two guide rails are mounted on the rail, together these components build the adapter plate. To reduce the calculation effort the mechanical cast component is modeled with a rigid body due to the larger flexibility of the carriage-rail system. Values of the spring stiffness of a whole carriage-rail system is extracted out of Data Sheets of the linear guidance manufacturer. Figure 3 depicts the model of the adapter plate with the four carriage-rail transitions. The usage of two spatial separated guide units

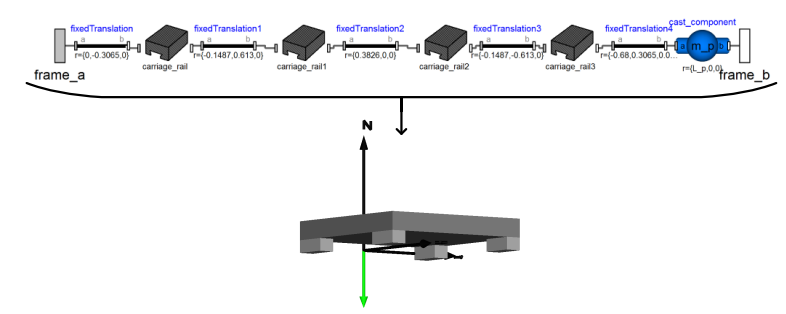

Figure 3. Model and animation of the adapter plate with four carriage-rail transitions

causes a distribution of the mechanical load and therefore an improved damping behaviour. The geometrical dimensions of the guide carriage and the spring stiffness will be presented to the carriage-rail transition models. Therefore this model especially characterises the transition between the fixed and the movable part of the machine, deformations of the components realizing this transition are neglected(Hoffmann, 2008). With the help of a compound slide, travel motions in y- and z-direction can be realised. Each of the two axis motions is equipped with two four-row linear recirculating ball bearings, where any of them consists of two carriage guides and one guide rail. The carriage rail system, which realises the z-motion is mounted at the back side of a steel plate in the same way as the adapter plate. A reverse arrangement, where the carriage-guides are mounted at the fixed part of the machine, which is the compound slide, provides movements in the y-direction. The carriage rails are fixed at the back side of the y-cantilever over the wohle dimension of the component. In the same way as the adapter plate is modeled, the compound slide will be modeled. Based on the assumption that the shift between the fixed and the movable parts is more flexible than the self deformation of the steel plate, only the carriage-rail systems are modeled flexibly. Figure 4 depicts the structure of the compound slide 
model with the eight carriage-rail transitions splitted into the different axis movements and the related 3D-model of the component. The animation of the component depicts

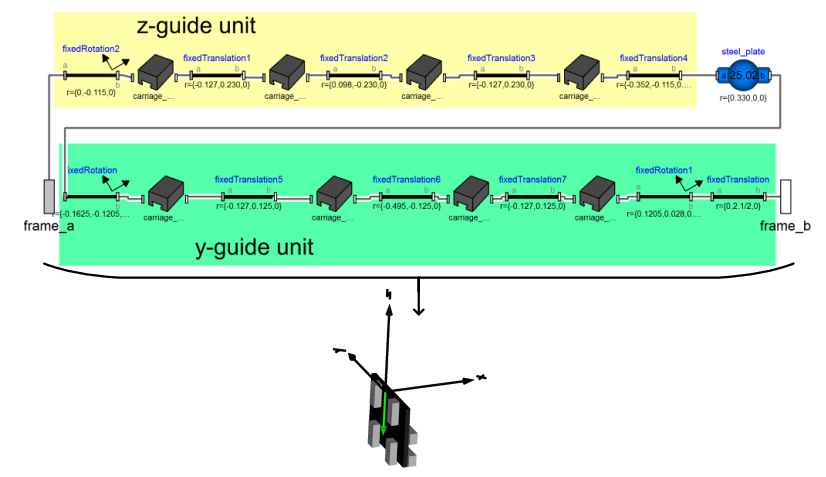

Figure 4. Model and animation of the compound slide with eight carriage-rail transitions splitted into the different axis movements

the reversal arrangement of the different linear guide systems. It makes no difference whether the carriage-rail shift between the y-cantilever and the compound slide is modeled at the slide itself or at the horizontal cantilever. In order to the same reasons as discussed at the adapter plate, only the flexibility of the transitions is modeled, the steel plate is assumed to be rigid.

\subsection{Modelling of the Milling Head}

The milling head is mounted at the end of the y-cantilever, it consists of eight parts. All of these parts are used to achieve rotary movements of the milling tool, which is mounted at the end of the component. Different connection flanges combine the rotary axes mechanically with each other. In comparision with the secondary and especially with the main machine parts the dimensions of the structural elements of the milling head are small. As a result of this assumption and as the milling head only acts as a load at the end of the y-cantilever the components can be modeled rigidly. In Figure 5 the model and the animation of the milling head is illustrated. The rotary motions of the different axes are implemented by components of the Modelica.Mechanics.Multibody.Joints li-

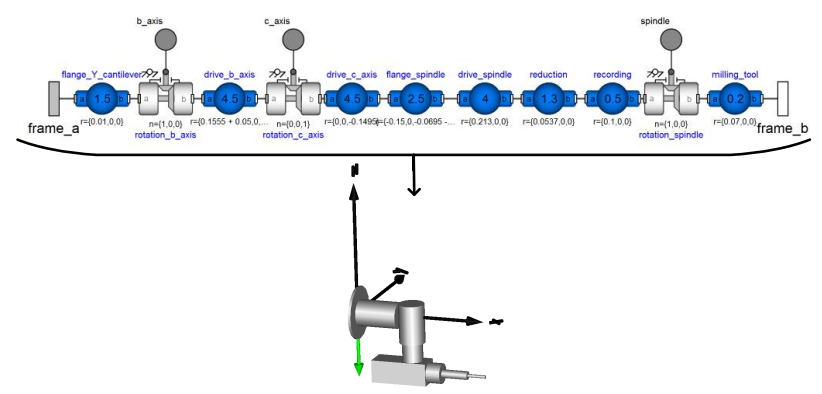

Figure 5. Model and animation of the milling head with rigid bodies brary. Different parameters of the rigid components, such as the mass or geometric dimensions, are defined by design data of the milling head.

\section{Modeling of the main machine parts}

\subsection{Modeling of the Y-Cantilever}

The initial situation at the y-cantilever can be described as follows, the cantilever is connected to the compound slide by the linear guides as described in section 3.2. It performs movements in the YZ-plane due to external forces caused by a linear direct drive. These forces, the connected milling head together with gravitational forces are leading to deformations of the canteliver and therefore to relative movements of the TCP with respect to the inital frame. For this reasons the y-cantilever has to be modeled with flexible bodies, and therefore the usage of the Flexiblebodies library is necessary. Choosing boundary conditions with respect to this situation would lead to the following conditions. In Figure 6 above, the initial situation with three choosen boundary conditions (red numbers) is depicted. The boundary conditions can be chosen

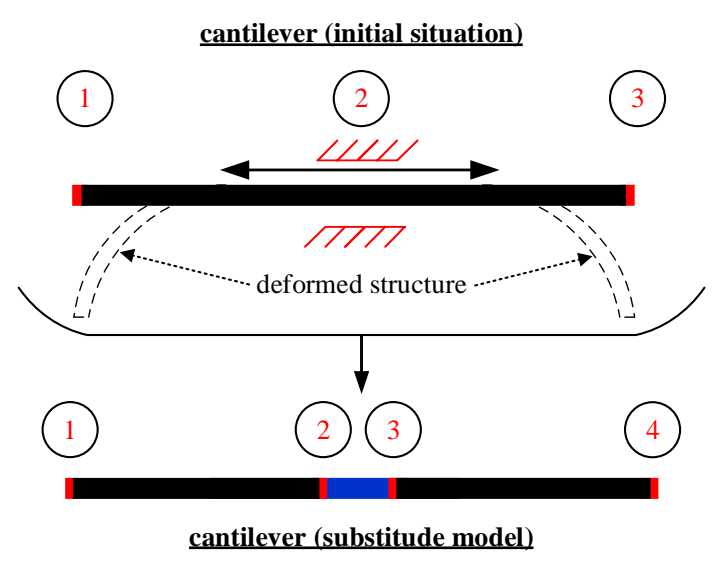

Figure 6. Inital situation of the y-cantilever and substituted model

free at point 1 and 3 and as movable support at number 2 because of the parallel guidance at the compound slide. The base class of a beam from the FlexibleBodies library enforces the user to choose boundary conditions for each deformation type at the endings of the beam to get correct results(Dr.-Ing. Andreas Heckmann et al., 2016). It is not possible to choose boundary conditions at certain points within the length of the beam. Choosing the base class of a beam model with two boundary conditions for each deformation type would therefore lead to large errors due to neglecting the third boundary condition. Furthermore the point, where the beam is movably supported changes during the milling process. In order to minimize the realtive error of the $y$-cantilever model a substitude model (Figure 6 bottom) has to be created. The model is splitted into three parts, where two parts consists of flexible beams (Figure 6 black) and one part is a rigid 
body (Figure 6 blue). The whole y-cantilever is divided at the center in two flexible beams of equal length. A rigid body, which describes the rigid clamping of the beam at the compound slide, is inserted between the flexible bodies. The division of the flexible structure and the insertion of a rigid body leads to a system where four boundary conditions have to be chosen but the conditions where the flexible beams are connected to the rigid body have to be clearly chosen as clamped. In order to align boundary conditions with the degrees of freedom of the joints, to which the beam is attached no condition should bound the elastic y-motion of the node attatched at the attachment frame(Heckmann, 2010). Because of the joint that realizes the $y$-motion of the beam, at point 1 no boundary condition is chosen for any type of deformation and the condition for point 4 is free. The restricition of this model is, that only small movements of the y-cantilever can be considered otherwise this model leads to massive errors. However the position of the y-cantilever can be assumed to be constant during the milling process of one part of a vehicle such as the side or the front surface. In addition to this problem parameters such as the cross section, the modulus of elasticity or the density can not be chosen easily. Taking a closer look at the cross section in Figure 7 of the flexible structures in the substituted model illustrates this fact. The entire beam consists of two parts,

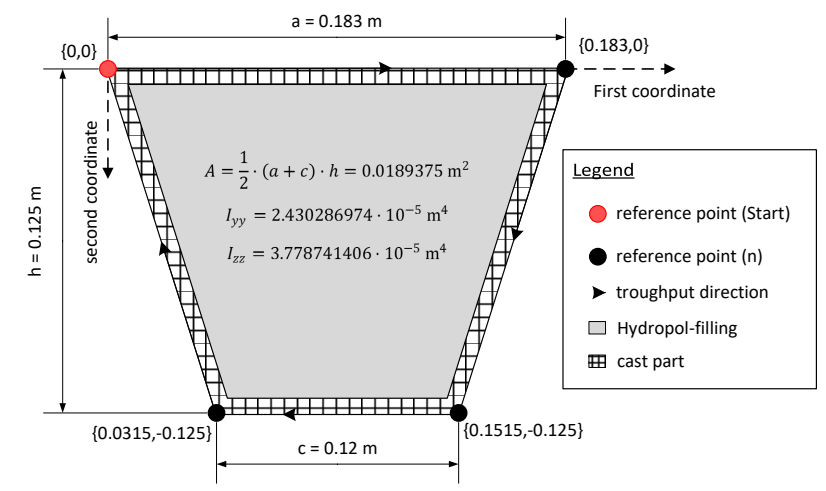

Figure 7. Cross section of the y-cantilever with geometrical moments of inertia

an empty cast part and a special vibration reducing material which is inserted there. The materials have different modulus of elasticity $E$ and different densities $\rho$, for this reason a fictitious material has to be developed, considering the shares $V_{f}$ of both materials. The material constants of the fictitious material can be calculated in the following way(Gross et al., 2014, p. 279-286).

$$
\begin{aligned}
V_{f} & =\frac{A_{\text {cast }}}{A_{\text {fill }}} \\
\rho_{\text {beam }} & =V_{f} \cdot \rho_{\text {cast }}+\left(1-V_{f}\right) \cdot \rho_{\text {fill }} \\
E_{\text {beam }} & =V_{f} \cdot E_{\text {cast }}+\left(1-V_{f}\right) \cdot E_{\text {fill }}
\end{aligned}
$$

Small movements of the y-cantilever are permissible but to consider the additional extension of the second beam, if it is moving towards the milling head, an additional line force has to be applied. This additive force has to be applied over the whole second beam and is depending on the covered distance towards the milling tool. The whole model of the y-cantilever with the additional line force acting as a point load and the animation of the component is depicted in Figure 8. In order to reduce dynamic flexi-

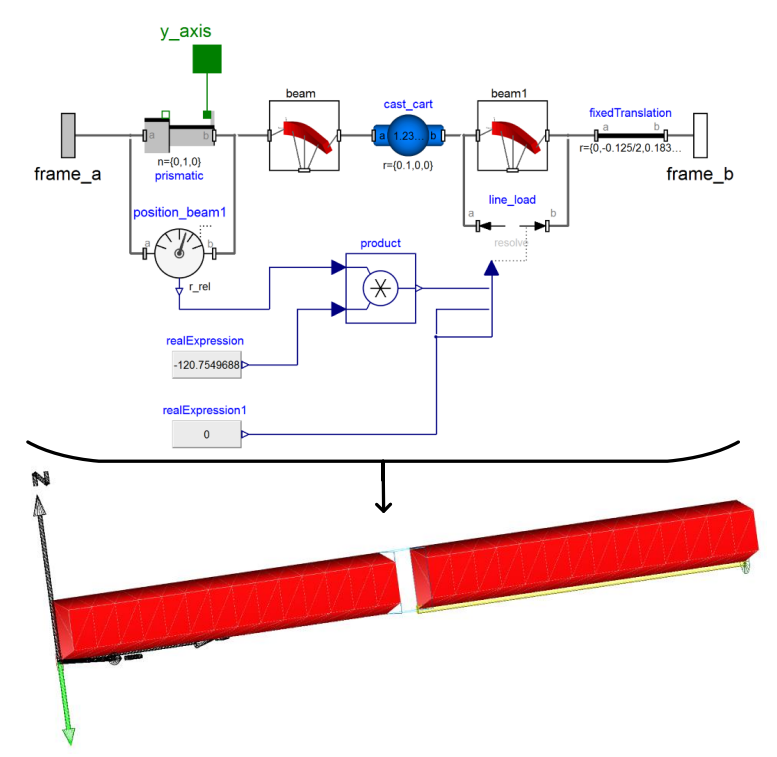

Figure 8. Model and animation of the y-cantilever with an additional line load

bility, steel ribs are welded over the whole length of the beam. This mechanical design detail could not be considered by the base class of a flexible beam without a subdivision of the y-cantilever model in several subsections and therefore a higher computational effort. Because of the new development of different machine parts, no real measurements are done on this components till yet. For this reason an independent validation of the y-cantilever model with the aid of real measurements is not possible.

\subsection{Modelling of the Z-Tower}

The second main machine part that shows high static and dynamic flexible behaviour is the z-tower. Due to the special geometry of this part, a simple beam model would not describe the behaviour. Therefore the second base class of the FlexibleBodies library, the ModalBody class is used to get more accurate results. A finite element model of the z-tower is reduced in two steps, in order to achieve a reduction of the number of degrees of freedom. The result of this reduction is a modal representation of the component with 221 degrees of freedom. This modal representation is stored in a standard input data file, that is the input for the ModalBody class either than for other multi-body simulation programs like SIMPACK. The second input file for the ModalBody provides informations of the geometri- 
cal shape of the considered body by a wavefront file. This file offers the user a 3D-view of the considered eg. the deformated component(Andreas Heckmann et al., p.88-94). In order to combine the movement of the compound slide and therefore the y-cantilever over the whole length of the tower the usage of the extended base class MovingLoad is suitable. It provides a connector, which allows it to attach moving forces/bodies/systems to the flexible structure. It is also possible to control this movement by a flange but additional parameter need to define this movement(Dr.Ing. Andreas Heckmann et al., 2016). Shape functions are used for defining deformations and calculating forces acting on the ModalBody, this shape functions are only known at certain points, specified by the Nodes parameter vector. This makes interpolation necessary and one requirement for the ModalBody especially it needs enough points in the vector to make this interpolation stable. The load is only connected to the right guide rail that is close to the milling head and the TCP. Six Simulation nodes out of 36 on this guide rail provide a stable interpolation. Another problem is to keep small the wavefront file, that provides a $3 \mathrm{D}$-view of the $\mathrm{z}$-tower otherwise the internal animation of Dymola will crash. The initial object file of the z-tower provided a mesh with 30095 elements. After applying a quadratic edge collapse decimaton filter a reduction to 1700 elements provides a stable animation. Figure 9 depicts the reduced animation file of the z-tower with the six simulaton nodes.

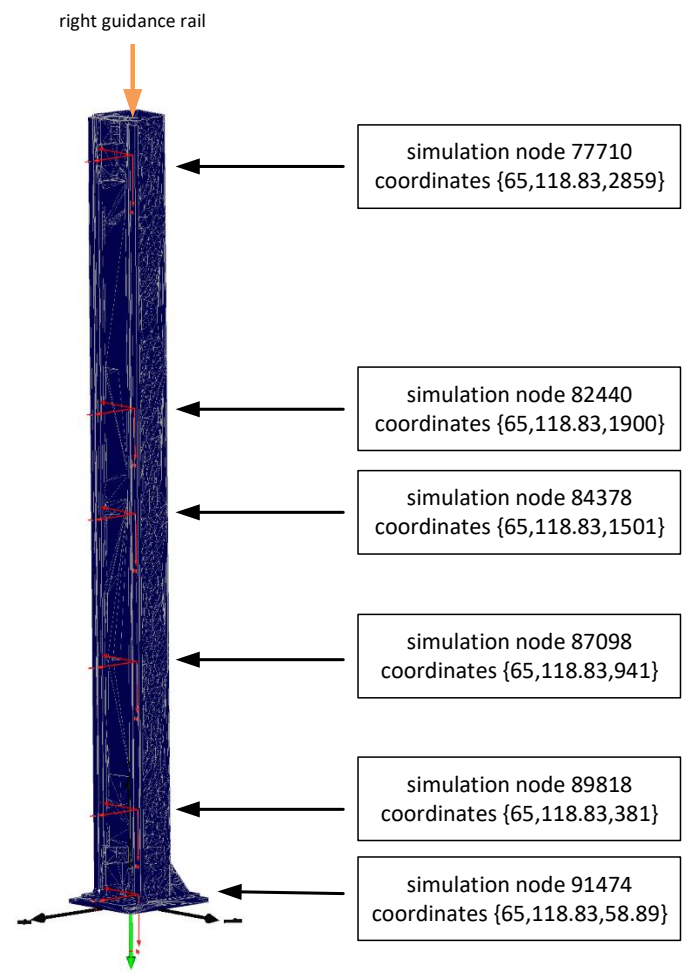

Figure 9. Reduced animation of the z-tower with used simualtion nodes and their coordinates

\section{Static and dynamic investigations}

The basis of the static and dynamic investigations are the presented secondary machine parts in section 3 and the main machine parts in section 4 . Together they build a multibody model with flexible and rigid bodies of the milling machine.

\subsection{Static investigations}

The static behaviour of the multibody model will be compared with the results of a finite element simulation in order to evaluate the multibody model in contrast to a mechanic specific simulation tool. The results of the finite element simulation are also not validated but they will match the real static behaviour of the machine close due to the integration of specific construction details out of CADconstructions. Both simulations are done without any load at the end of the y-cantilever. To evaluate the static behaviour of the model a constant force in one direction is applied at the end of the $y$-cantilever to get static shifts in all three translational directions. This constant force will be achieved through a step function with a short delay time to provide that the whole structure is in a steady state. The $y$-cantilever will therefore be in an unstable position at the highest point at the z-tower and fully extended. The area in which the substitute model of the y-cantilever is valid is left and only the magnitudes of the static shifts can be rated. After five seconds to get the structure in a steady state, a constant force of $F=10 \mathrm{~N}$ is applied at the end of the y-cantilever and after $0,35 \mathrm{~s}$ the structure comes already to a steady state. If the relative movement at the

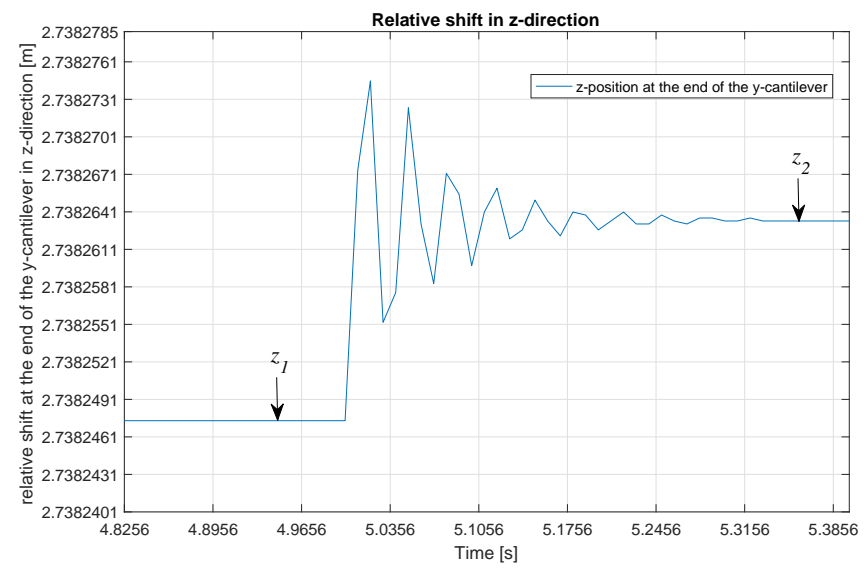

Figure 10. Relative shift at the end of the y-cantilever in zdirection

end of the $y$-cantilever is divided by the applied force the relative shift $V_{z}$ in $\mathrm{z}$-direction can be calculated.

$$
V_{z}=\frac{z_{2}-z_{1}}{F}=\frac{1.5974 \cdot 10^{-5} \mathrm{~m}}{10 \mathrm{~N}}=1.5974 \cdot 10^{-3} \frac{\mathrm{mm}}{\mathrm{N}}
$$


In the same way as shown above the relative shifts in yand $\mathrm{x}$-direction can be calculated.

$$
\begin{aligned}
& V_{y}=\frac{y_{2}-y_{1}}{F}=\frac{1.0729 \cdot 10^{-5} \mathrm{~m}}{10 \mathrm{~N}}=1.0729 \cdot 10^{-3} \frac{\mathrm{mm}}{\mathrm{N}} \\
& V_{x}=\frac{x_{2}-x_{1}}{F}=\frac{5.9605 \cdot 10^{-6} \mathrm{~m}}{10 \mathrm{~N}}=5.9605 \cdot 10^{-4} \frac{\mathrm{mm}}{\mathrm{N}}
\end{aligned}
$$

Comparing the results of the multibody simulation to the finite element solutions it can be stated that in a good approximation they are convergent. Deviations can be justified by the neglection of the flexibility of the guidance system, the disregardence of the ribs in the y-cantilever and the desertion of the scope of the y-cantilever. Regarding the magnitudes of the static shifts it can be said that they are absolutely convergent to the finite element solution.

\subsection{Dynamic investigations}

Positioning movements will encourage oscillations of the whole structure and therefore also vibrations of the TCP. This oscilations will also lead to positioning errors during the milling process. To have a closer look at a positioning movement the y-cantilever will be in a stable position in the centre of the traverse range of the z-tower. A simple ramp function will increase the x-position of the structure till reaching a final value. The substitude model of the y-cantilever is therefore in position that is within the validity range. The ramp starts at position $x=0 \mathrm{~m}$ at $0.5 \mathrm{~s}$ and increases this position till reaching the final value of $x=1 \mathrm{~m}$ at $1.5 \mathrm{~s}$. Figure 11 depicts the dynamical behaviour during the positioning process. The simula-

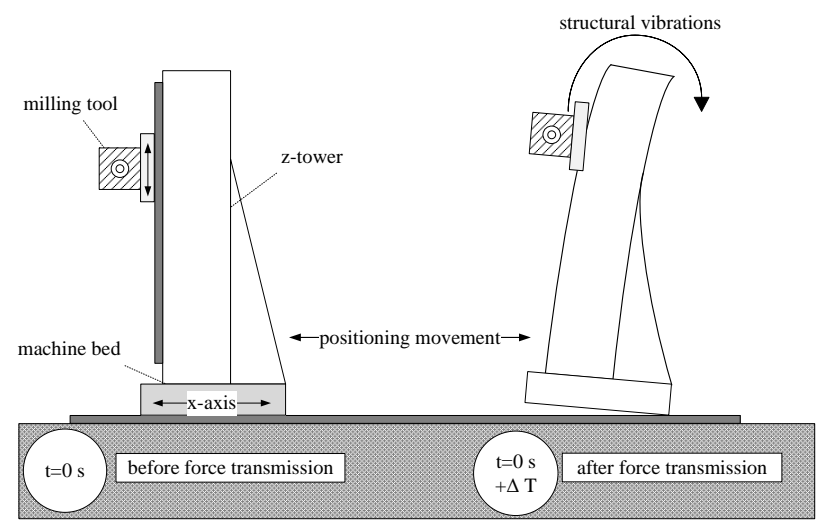

Figure 11. Dynamical behaviour during the positioning movements

tion results in Figure 12 are showing absolutely the same behaviour of the structure. After reaching the final position an overshoot relative to the desired position could be determined. The envelope of this overshoot is an exponential function which indicates a strong viscose damping behaviour of the structure(Hoffmann, 2008).

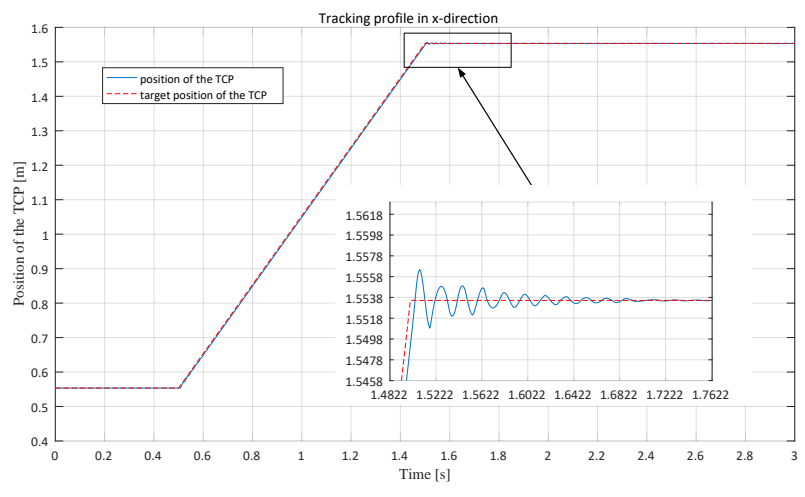

Figure 12. Tracking profile in $\mathrm{x}$-direction

\section{Conclusion and outlook}

The mechanical model of the 5-axis-milling machine depicts the static and dynamic behaviour very accurate. The simplifications made during the modeling process have only marginal influences on the result. For modeling flexible bodies within multibody structures the FlexibleBodies library is a very accurate tool. The base classes Beam and ModalBody offers the user different methods of implementing those flexible structures. The beam class is only useful for describing simple beam models, complex geometries or designs are difficult to model with this class. An extension of the beam base class, where the user is able to define more than two boundary conditions and make the position of these boundary conditions time dependent would therefore be a relief. The amount of usable boundary conditions is limited to three, implementing other boundary conditions such as "movable clamped" would make the modeling of problems, such as the movable cantilever, easier without FE-pre-processing. In order to model such beam structures and defining input datas such as boundary conditions or the number of the required eigenmodes for getting correct results, a very deep theoretical background is needed. If the user only wants to implement existing models derived out of a finite element analysis the ModalBody class offers a very powerful tool for implementing those structures. In the next step, models of the drive trains that move the different axes will be developed. In combination with this drive models the whole behaviour of the mechatronic system can be observed and compared to the real system. After this validation of the model the trajectory guidance will be improved by changing the control strategy of the drive trains.

\section{References}

Andreas Heckmann, Martin Otter, Stefan Dietz, and José Díaz López. The DLR FlexibleBodies library to model large motions of beams an of flexible bodies exported from finite element programs. In Proceedings of the 5th International Modelica Conference, pages 85-95. URL https://www.modelica.org/events/ 
modelica2006/Proceedings/proceedings /

Proceedings2006_Vol1.pdf.

Dr.-Ing. Andreas Heckmann, Prof. Dr.-Ing. Martin Otter, Martin Leitner, Jakub Tobolar, and Stefan Hartweg. FlexibleBodies: Library to model large motions of flexible beams, anular plates and of flexible bodies exported from finite element programs, 2016. Version 2.2.

Dietmar Gross, Werner Hauger, Jörg Schröder, and Wolfgang A. Wall. Technische Mechanik 2: Elastostatik. SpringerLehrbuch. Springer Vieweg, Berlin, 12., aktual. aufl. edition, 2014. ISBN 978-3-642-40965-3. doi:10.1007/978-3642-40966-0. URL http://dx.doi.org/10.1007/ 978-3-642-40966-0.

Andreas Heckmann. On the choice of boundary conditions for mode shapes in flexible multibody systems. Multibody System Dynamics, 23(2):141-163, 2010. ISSN 1384-5640. doi:10.1007/s11044-009-9177-z.

Frank Hoffmann. Optimierung der dynamischen Bahngenauigkeit von Werkzeugmaschinen mit der Mehrkörpersimulation: Zugl.: Aachen, Techn. Hochsch., Diss., 2008, volume 2008,8 of Ergebnisse aus der Produktionstechnik Werkzeugmaschinen. Apprimus-Verl., Aachen, 2008. ISBN 978-3940565-12-9.

Marcus Queins. Simulation des dynamischen Verhaltens von Werkzeugmaschinen mit Hilfe flexibler Mehrkörpermodelle: Zugl.: Aachen, Techn. Hochsch., Diss., 2005, volume 2005,12 of Berichte aus der Produktionstechnik. Shaker, Aachen, 2005. ISBN 3-8322-4224-4. 